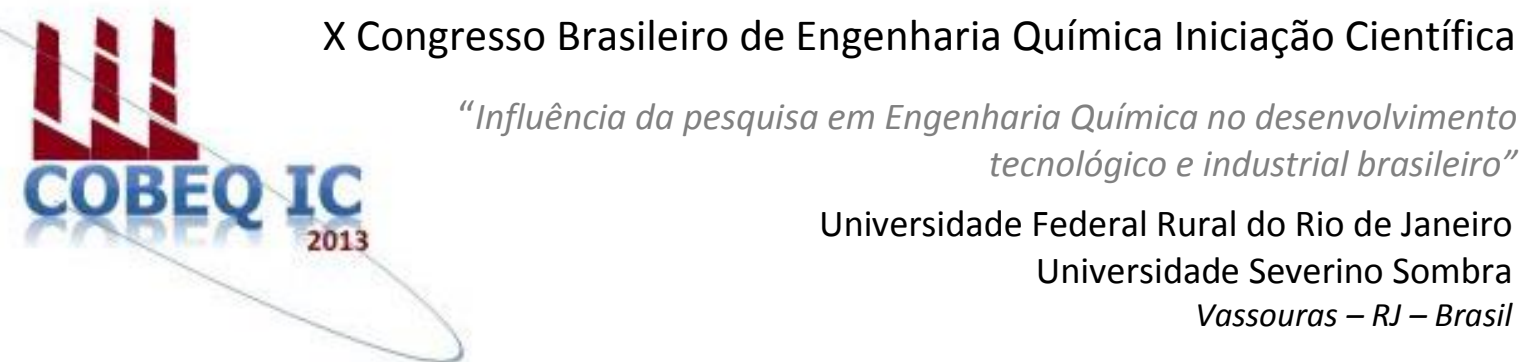

\title{
ESTUDO DAS ISOTERMAS DE ADSORÇÃO DE AZO-CORANTES POR FILMES DE QUITOSANA
}

\author{
CAETANO $^{* 1}$, C. M. B.; LOPES ${ }^{* * 1}$, A. C.; RÊGO ${ }^{2}$, T. V.; DOTTO ${ }^{3}$, G. L.; PINTO**4 L. A. A. \\ ${ }^{1}$ Aluno do EQA/FURG $\quad{ }^{2}$ Mestrando do PPG-ECA/FURG $\quad{ }^{3}$ Professor DEQ/UFSM ${ }^{4}$ Professor \\ do PPG-ECA/FURG \\ Escola de Química e Alimentos - Universidade Federal de Rio Grande \\ Endereço-FURG, Rua Eng. Alfredo Huch, 475, 96203-900, Rio Grande, RS, CEP. 96201-900, RS, \\ email: dqmpinto@ furg.br \\ Departamento de Engenharia Química - Universidade Federal de Santa Maria \\ Endereço-UFSM, Av. Roraima, 1000, Santa Maria, RS, 97105-900.
}

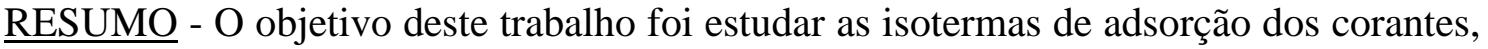
amaranto e tartrazina por filmes de quitosana. Os filmes foram preparados pela técnica casting e caracterizados. Os experimentos foram realizados nas seguintes condições: concentração inicial de corante na faixa de 50-300 mg L $\mathrm{m}^{-1}$, concentração inicial de filme de $100 \mathrm{mg} \mathrm{L}^{-1}$, pH 2, sendo verificado o efeito da temperatura (298-328 K). Para avaliar as curvas de equilíbrio foram utilizados os modelos de isoterma de Langmuir e Freundlich. Os filmes de quitosana apresentaram espessura inicial de $62 \pm 3 \mu \mathrm{m}$, tensão

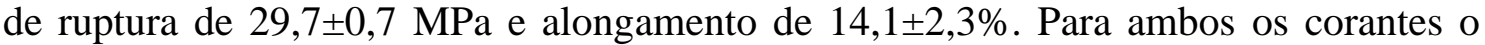
aumento da temperatura afetou negativamente a capacidade de adsorção. O modelo de Langmuir foi o mais adequado para representar os dados experimentais. Os valores de $\mathrm{k}_{\mathrm{L}}$ e $\mathrm{q}_{\mathrm{m}}$ aumentaram com a diminuição da temperatura, mostrando que a adsorção foi favorecida à $298 \mathrm{~K}$. Foi verificado que as capacidades máximas de adsorção aumentaram com a diminuição da temperatura, alcançando valores máximos de 494,1 e $480,0 \mathrm{mg} \mathrm{g}^{-1}$ para os corantes amaranto e tartrazina, respectivamente.
\end{abstract}

Palavras chave: amaranto, Langmuir, tartrazina.

\section{INTRODUÇÃO}

Estima-se que existam no mundo entre corantes sintéticos e pigmentos, aproximadamente 10000 tipos (Mahmoodi et al., 2008). No Brasil segundo ANVISA (1978), onze são os corantes permitidos para alimentos entre estes, temos o amaranto e o amarelo tartrazina pertencentes à classe dos azo compostos. Os corantes dessa classe são considerados tóxicos e, estudos afirmam que estes podem tornar-se cancerígenos ao se reduzir em aminas aromáticas pela microflora intestinal humana, além de causar outros problemas como asma, eczema e hiperatividade em crianças (Moutinho et al., 2007). Outro inconveniente está no excesso de efluentes gerado durante o processamento de alimentos pelas indústrias. Estes são prejudiciais ao ambiente principalmente

*Bolsista Permanência FURG; ** Bolsista Voluntária FURG. 
devido a sua toxidade e a possível alteração do sistema aquático, logo, torna-se extremamente importante $\mathrm{o}$ adequado tratamento destes efluentes (Crini e Badot, 2008).

A adsorção tem se mostrado como um método alternativo para o tratamento de efluentes coloridos, principalmente quando se utilizam adsorventes de baixo custo (Gupta e Suhas, 2009). Entre os biopolímeros estudados, a quitosana apresenta-se como um material promissor para fins de adsorção (Wibowo et al., 2007). A quitosana é versátil além de possuir um bom custo benefício em relação a outros biopolímeros (Crini e Badot, 2008). Entre os vários materiais a base de quitosana utilizados, os filmes apresentam destaque na adsorção de corantes principalmente devido a suas capacidades mecânicas além da sua possibilidade de separação pós-processo de adsorção (Fajardo et al., 2012).

No que tange a adsorção de corantes por filmes de quitosana, ainda são necessários muitos estudos para uma melhor compreensão e entendimento do processo. O estudo das isotermas de equilíbrio é fundamental, uma vez que, sua forma auxilia no esclarecimento do tipo de mecanismo envolvido na adsorção. Além disso, os parâmetros de equilíbrio fornecem informações sobre a qualidade do adsorvente e a afinidade adsorvente-adsorbato (Blázquez et al., 2010).

Sendo assim, o objetivo deste trabalho foi estudar as isotermas de adsorção dos corantes, amaranto e tartrazina por filmes de quitosana. Foi verificado o efeito da temperatura e os modelos de Langmuir e Freundlich foram ajustados aos dados experimentais.

\section{MATERIAL E MÉTODOS}

\section{Adsorbato}

Os corantes foram fornecidos pela Plury Química Ltda., (Brasil) com grau de pureza de $85 \%$. O corante amarelo tartrazina possui índice de cor de 19140, massa molar de 534,4 $\mathrm{g} \mathrm{mol}^{-1}$ e comprimento de onda de máxima absorção de $425 \mathrm{~nm}$. O corante amaranto possui índice de cor de 16185, massa molar de 604,5 $\mathrm{g} \mathrm{mol}^{-1}$ e comprimento de onda de máxima absorção de $521 \mathrm{~nm}$. As estruturas químicas dos corantes amarelo tartrazina e vermelho amaranto estão apresentadas nas Figuras 1 e 2, respectivamente.

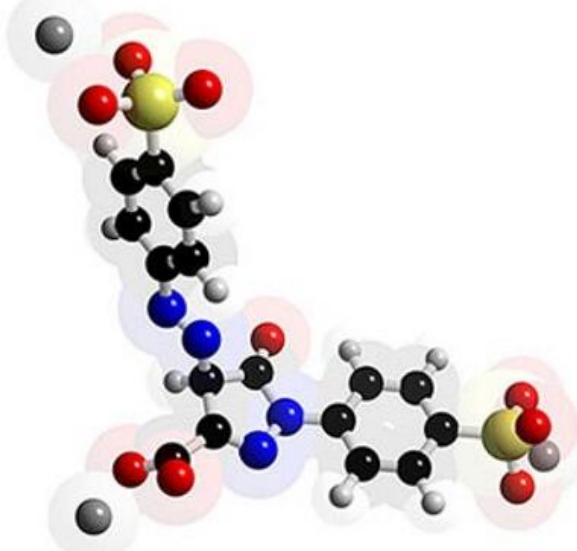

Figura 1 - Estrutura química do corante amarelo tartrazina

(Fonte: www.3dchem.com).

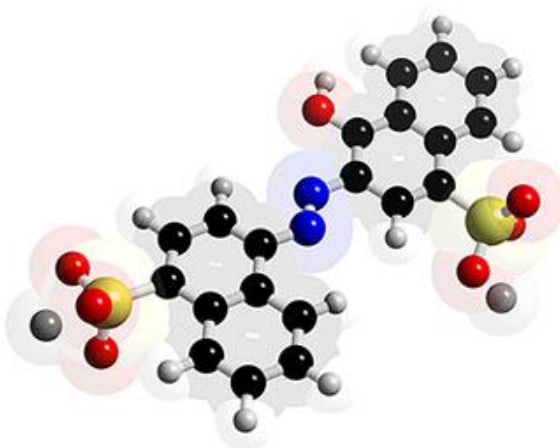

Figura 2- Estrutura química do corante amaranto (Fonte: www.3dchem.com).

\section{Obtenção da quitosana}

A quitosana foi obtida a partir de resíduos de camarão (Penaeus brasiliensis). Os resíduos passaram pelas etapas de lavagem, desmineralização, desproteinização, desodorização e secagem obtendo a quitina seca; após realizou-se a desacetilação com solução de hidróxido de sódio $(42,1 \%$ p/v) a $130 \pm 2^{\circ} \mathrm{C}$, por 90 min com agitação mecânica de $50 \mathrm{rpm}$. A quitosana obtida foi purificada de acordo com Weska et al., (2007). A secagem da pasta de quitosana foi realizada de acordo com Dotto et al. (2011), obtendo-se um pó com $72 \pm 3 \mu \mathrm{m}$ de diâmetro.

\section{Preparação dos filmes}

As amostras de quitosana em pó (1,5g, base seca) foram dissolvidas em $50 \mathrm{~mL}$ de 
uma solução $0,1 \mathrm{~mol} \mathrm{~L}^{-1}$ de ácido acético. Após, procedeu-se agitação magnética por 24 $\mathrm{h}$ à temperatura ambiente. A solução filme formada foi filtrada, colocada em placas de Petri (14 cm de diâmetro) e seca a $40{ }^{\circ} \mathrm{C}$, em estufa com circulação de ar por $48 \mathrm{~h}$ para a evaporação do solvente. Os filmes foram armazenados em dessecadores (Dotto et al., 2013).

\section{Caracterização dos filmes}

Os filmes foram caracterizados em relação à tensão de ruptura e alongamento (ASTM, 2001), utilizando um analisador de textura (Stable Micro Systems, TA-XT-2i, Reino Unido). A espessura foi determinada utilizando-se um paquímetro digital (Stainless Hardened, $150 \mathrm{~mm}$, China) com resolução de $0,001 \mathrm{~mm}$.

\section{Experimentos de equilíbrio de adsorção}

Os experimentos foram realizados em batelada em um agitador termostatizado tipo Wagner (Fanem, 315 SE, Brasil) nas seguintes condições: concentração inicial de filme de $100 \mathrm{mg} \mathrm{L}^{-1}, \mathrm{pH} 2$ e taxa de agitação $100 \mathrm{rpm}$. As temperaturas variaram de 298, 308, 318 e $338 \mathrm{~K}$ e a concentração inicial variou de 50 a $300 \mathrm{mg} \mathrm{L}^{-1}$. Alíquotas foram retiradas no equilíbrio, sendo determinada a concentração dos corantes por espectrofotometria (Quimis, 108 DRM, Brasil) à 426 e $521 \mathrm{~nm}$ para a tartrazina e amaranto respectivamente. A capacidade de adsorção no equilíbrio $\left(\mathrm{q}_{\mathrm{e}}\right)$ foi calculada de acordo com a Equação 1:

$$
\mathrm{q}_{\mathrm{e}}=\frac{\mathrm{C}_{0}-\mathrm{C}_{\mathrm{e}}}{\mathrm{m}} \mathrm{V}
$$

\section{Modelos de equilíbrio}

Para o ajuste dos dados experimentais de equilíbrio foram aplicados os modelos de isotermas de Langmuir e Freundlich, que estão apresentados nas Equações 2 e 3, respectivamente (Crini e Badot, 2008):

$$
\begin{aligned}
& \mathrm{q}_{\mathrm{e}}=\frac{\mathrm{q}_{\mathrm{m}} \mathrm{k}_{\mathrm{L}} \mathrm{C}_{\mathrm{e}}}{1+\mathrm{k}_{\mathrm{L}} \mathrm{C}_{e}} \\
& \mathrm{q}_{\mathrm{e}}=\mathrm{k}_{\mathrm{f}} \mathrm{C}_{\mathrm{e}}^{1 / \mathrm{n}}
\end{aligned}
$$

Os parâmetros das isotermas foram estimados pelo ajuste das Equações 2 e 3 com os dados experimentais, utilizando regressão não linear. Foi utilizado o método de estimação Quasi-Newton. Os cálculos foram realizados com auxílio do software Statistic 7.0 (Statsoft, EUA), e qualidade dos ajustes foi mensurada de acordo com o coeficiente de determinação $\left(\mathrm{R}^{2}\right)$ e o erro médio relativo (EMR) (El-Khaiary e Malash, 2011).

\section{RESULTADO E DISCUSSÃO}

\section{Características dos filmes}

Os filmes elaborados de quitosana apresentaram tensão de ruptura de 29,7 $\pm 0,7$ $\mathrm{MPa}$, espessura inicial de $62 \pm 3 \mu \mathrm{m}$ e alongamento de $14,1 \pm 2,3 \%$. Estes valores foram similares aos obtidos por Moura et al., (2011) e Rêgo et al., (2013). Os filmes de quitosana com essas propriedades são considerados adequados para aplicação na adsorção dos corantes.

\section{Isotermas de equilíbrio}

As curvas de equilíbrio de adsorção dos corantes estão apresentadas nas Figuras 3 e 4.

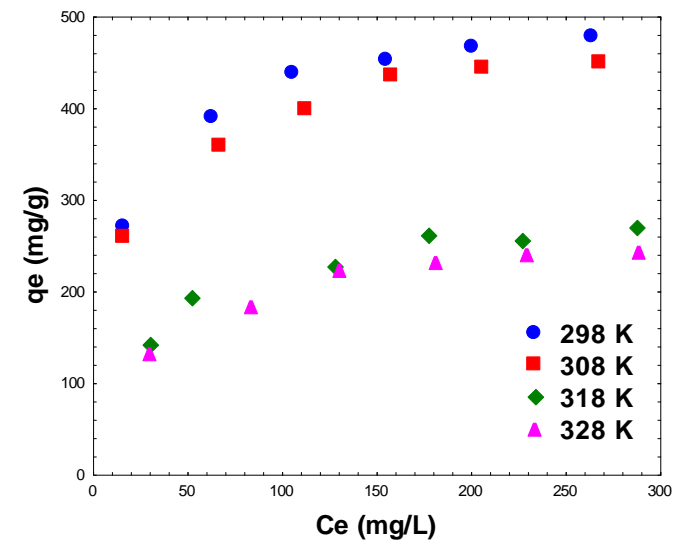

Figura 3 - Curvas de equilíbrio na adsorção do corante amaranto por filmes de quitosana. 


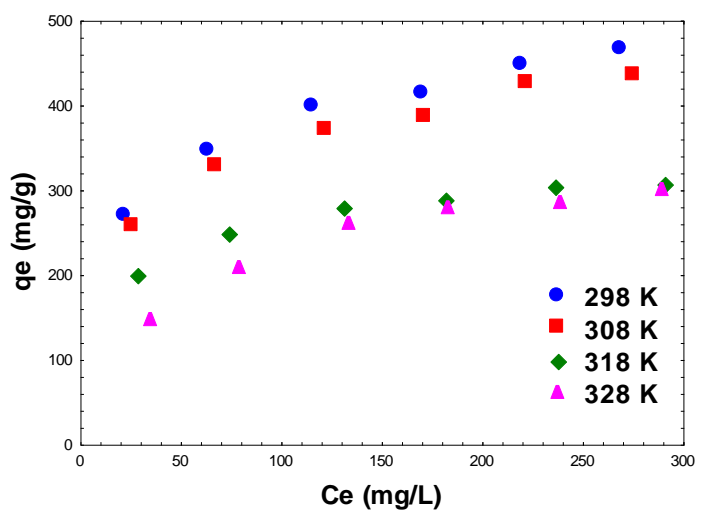

Figura 4 - Curvas de equilíbrio na adsorção do corante amarelo tartrazina por filmes de quitosana.

Nas Figuras 3 e 4 verifica-se que as isotermas são caracterizadas como sendo do tipo "L" (Giles et al., 1960). Verificou-se uma etapa inicial de aumento na capacidade de adsorção, a qual indica uma boa afinidade entre o filme de quitosana e os azo corantes, seguida de um platô, que representa a máxima capacidade de saturação. Observa-se também, que com o aumento da temperatura ocorreu uma diminuição na capacidade de adsorção.
Isto é devido ao fato de que o aumento da temperatura leva a um aumento da solubilidade dos corantes aniônicos (Crini e Badot, 2008), fazendo com que estes aumentem sua preferência pela água em relação ao adsorvente.

A fim de obter mais informações em relação às isotermas, os dados de equilíbrio foram ajustados aos modelos de Langmuir e Freundlich, e os resultados dos ajustes estão apresentados nas Tabelas 1 e 2 .

Os altos valores do coeficiente de determinação $\left(\mathrm{R}^{2}>0,99\right)$ e os baixos valores do erro médio relativo $(\mathrm{E}<2,5 \%)$ apresentados na Tabela 1, demonstram que o modelo de isoterma de Langmuir foi o que apresentou o melhor ajuste aos dados experimentais de equilíbrio. Os valores do parâmetro $\mathrm{q}_{\mathrm{m}}$ (Tabela 1) aumentaram em função da diminuição da temperatura, mostrando que a capacidade de adsorção foi favorecida a $298 \mathrm{~K}$. Os valores de $\mathrm{k}_{\mathrm{L}}$ apresentaram a mesma tendência, indicando que a afinidade dos filmes com os azo corantes foi favorecida pela diminuição da temperatura.

Tabela 1 - Parâmetros do modelo de Langmuir para a adsorção dos corantes.

\begin{tabular}{ccccccccc}
\hline & \multicolumn{4}{c}{ Amaranto } \\
\cline { 2 - 9 } & $\mathrm{k}_{\mathrm{L}}$ & $\mathrm{q}_{\mathrm{m}}$ & $\mathrm{R}^{2}$ & $\mathrm{EMR}$ & $\mathrm{k}_{\mathrm{L}}$ & $\mathrm{q}_{\mathrm{m}}$ & $\mathrm{R}^{2}$ & $\mathrm{EMR}$ \\
\cline { 2 - 9 } 298 & 0,072 & 494,1 & 0,9979 & 1,39 & 0,049 & 480,0 & 0,9915 & 1,03 \\
308 & 0,066 & 465,7 & 0,9959 & 1,97 & 0,045 & 453,4 & 0,9918 & 1,09 \\
318 & 0,031 & 297,2 & 0,9947 & 2,48 & 0,050 & 324,4 & 0,9983 & 1,37 \\
328 & 0,028 & 273,5 & 0,9952 & 2,32 & 0,019 & 351,5 & 0,9983 & 1,42 \\
\hline
\end{tabular}

Tabela 2 - Valores obtidos pelo modelo de Freundlich.

\begin{tabular}{ccccccccc} 
& \multicolumn{9}{c}{ Amaranto } \\
\cline { 2 - 9 } & $\mathrm{k}_{\mathrm{F}}$ & $1 / \mathrm{n}$ & $\mathrm{R}^{2}$ & $\mathrm{EMR}$ & $\mathrm{k}_{\mathrm{F}}$ & $1 / \mathrm{n}$ & $\mathrm{R}^{2}$ & EMR \\
\cline { 2 - 9 } 298 & 171,9 & 5,3 & 0,9930 & 2,93 & 140,8 & 4,7 & 0,9906 & 1,18 \\
308 & 152,3 & 5,0 & 0,9957 & 1,97 & 129,6 & 4,6 & 0,9908 & 1,20 \\
318 & 65,2 & 3,9 & 0,9871 & 4,15 & 110,5 & 5,4 & 0,9973 & 1,58 \\
328 & 56,8 & 3,8 & 0,9874 & 3,51 & 52,4 & 3,2 & 0,9895 & 3,61 \\
\hline
\end{tabular}

As máximas capacidades de adsorção $\left(\mathrm{q}_{\mathrm{m}}\right)$ foram de 494,1 e 480,0 $\mathrm{mg} \mathrm{g}^{-1}$ para os corantes amaranto e tartrazina, respectivamente, obtidos a $298 \mathrm{~K}$ (Tabela 1). A literatura mostra que o valor de $\mathrm{q}_{\mathrm{m}}$ em sistemas de adsorção de corantes por quitosana pode variar entre $50 \mathrm{mg}$ $\mathrm{g}^{-1}$ e $2500 \mathrm{mg} \mathrm{g}^{-1}$ (Crini e Badot, 2008). Em 
vista disso pode-se afirmar que a quitosana na forma de filmes utilizada neste trabalho possui uma boa capacidade de adsorção.

\section{CONCLUSÃO}

Neste trabalho, foram estudadas as isotermas de equilíbrio de adsorção dos corantes amaranto e tartrazina, utilizando filmes de quitosana como adsorvente. Os resultados mostraram que a diminuição da temperatura da temperatura favoreceu a adsorção, e as isotermas foram caracterizadas como do tipo "L". O modelo de Langmuir foi o mais adequado para representar os dados experimentais. Os valores de $\mathrm{q}_{\mathrm{m}}$ aumentaram com a diminuição da temperatura, alcançando valores máximos de 494,1 e 480,0 $\mathrm{mg} \mathrm{g}^{-1}$ para os corantes amaranto e tartrazina, respectivamente, a $298 \mathrm{~K}$.

\section{NOMENCLATURA}

\begin{tabular}{|c|c|c|}
\hline$\left[\mathrm{C}_{0}\right]$ & $\begin{array}{c}\text { Concentração inicial de } \\
\text { corante }\end{array}$ & {$\left[\mathrm{mg} \mathrm{L}^{-1}\right]$} \\
\hline [EMR] & Erro médio relativo & [\%] \\
\hline [m] & Massa de adsorvente & {$[\mathrm{g}]$} \\
\hline$[\mathrm{V}]$ & Volume de solução & [L] \\
\hline$\left[\mathrm{q}_{\mathrm{e}}\right]$ & $\begin{array}{l}\text { Capacidade de } \\
\text { adsorção no equilíbrio }\end{array}$ & {$\left[\mathrm{mg} \mathrm{L}^{-1}\right]$} \\
\hline$\left[\mathrm{q}_{\mathrm{m}}\right]$ & $\begin{array}{c}\text { Capacidade de } \\
\text { adsorção máxima }\end{array}$ & {$\left[\mathrm{mg} \mathrm{L}^{-1}\right]$} \\
\hline$\left[\mathrm{C}_{\mathrm{e}}\right]$ & $\begin{array}{l}\text { Concentração no } \\
\text { equilibrio }\end{array}$ & {$\left[\mathrm{mg} \mathrm{L}^{-1}\right]$} \\
\hline$\left[\mathrm{k}_{\mathrm{L}}\right]$ & Constante de Langmuir & {$\left[\mathrm{L} \mathrm{mg}^{-1}\right]$} \\
\hline$\left[\mathrm{k}_{\mathrm{F}}\right]$ & $\begin{array}{l}\text { Constante de } \\
\text { Freundlich }\end{array}$ & $\begin{array}{c}{\left[\left(\mathrm{mg} \mathrm{g}^{-1}\right) /\right.} \\
\left.\left(\mathrm{m} \mathrm{L}^{-1}\right)\right]\end{array}$ \\
\hline $\begin{array}{c}\mathrm{R}^{2} \\
{[1 / \mathrm{n}]}\end{array}$ & $\begin{array}{c}\text { Coef. de determinação } \\
\text { Fator de } \\
\text { heterogeneidade }\end{array}$ & {$\left[\mathrm{mg} \mathrm{L}^{-1}\right]$} \\
\hline
\end{tabular}

\section{REFERÊNCIAS}

ASTM (2001) Standard test method for tensile properties of thin plastic sheeting. Designation D882. In Annual Book of American Standard Testing Methods (pp. 162-170). Philadelphia: American Society for Testing and Materials.
BLÁZQUEZ, G., CALERO, M., HERNÁINZ, F., TENORIO, G., MARTÍN-LARA, M.

A. (2010), Equilibrium biosorption of lead (II) from aqueous solutions by solid waste from olive-oil production. Chemical Engineering Journal, 160, 615-622.

BRASIL. ANVISA. (1978), Resolução CNNPA $\mathrm{n}^{\circ} 44$ de 1978. DOU de 28/04/1978- Disponível em: http://www.anvisa.gov.br.

CHEMISTRY, STRUCTURES \& 3D MOLECULES @3Dchem.com - Home. Disponível em http://www.3dchem.com.

CRINI, G., BADOT, P. M. (2008), Application of chitosan, a natural aminopolysaccharide, for dye removal from aqueous solutions by adsorption processes using batch studies: A review of recent literature. Progress in Polymer Science, 33, 399-447.

DOTTO, G. L., MOURA, J. M., CADAVAL, T. R. S., PINTO, L. A. A. (2013), Application of chitosan films for the removal of food dyes from aqueous solutions by adsorption. Chemical Engineering Journal, 214, 8-16.

DOTTO, G. L., SOUZA, V. C., MOURA, J. M., MOURA, C. M., PINTO, L. A. A. (2011), Influence of drying techniques on the characteristics of chitosan and the quality of biopolymer films. Drying Technology, 29, 1784-1791.

FAJARDO, A. R., LOPES, L. C., RUBIRA, A. F., MUNIZ, E. C. (2012), Development and application of chitosan/poly(vinyl alcohol) films for removal and recovery of $\mathrm{Pb}$ (II). Chemical Engineering Journal, 183, 253-260.

GILES, C. H., MACEWAN, T. H., NAKHWA, S. N., SMITH, D. (1960), Studies in adsorption part XI: A system of classification of solution adsorption isotherms and its use in diagnosis of adsorption mechanisms and in measurement of specific surface areas of solids. Journal of Chemical Society 39733993.

GUPTA, V. K., SUHAS. (2009), Application of low-cost adsorbents for dye removal: A review. Journal of Environmental Management, 90, 2313. 
EL-KHAIARY, M. I., MALASH, G. F. (2011), Common data analysis errors in batch adsorption studies.

Hydrometallurgy, 105, 314-320.

MAHMOODI, N.M. HAYATI, B. ARAMI, M. LAN, C. (2011), Adsorption of textile dyes on pine cone from colored wastewater: Kinetic, equilibrium and thermodynamic studies. Desalination 268, 117-125.

MOURA, C. M., MOURA, J. M., SOARES, N. M., PINTO, L. A. A. (2011), Evaluation of molar weight and deacetylation degree of chitosan during chitin deacetylation reaction: used to produce biofilm. Chemical Engineering and Processing: Process Intensification, 50, 351-355.

MOUTINHO, I. L., BERTGES, L. C., ASSIS, R. V. (2007), Prolonged use of the dye Tartrazine (FD\&C yellow $\mathrm{n}^{\circ}$ 5) and its effects on the gastric mucosa of Wistar rats. Brazilian Journal of Biology: 67(1), 141-145.

RÊGO, T. V., CADAVAL JR., T. R. S., DOTTO, G. L., PINTO, L. A. A. (2013), Statistical optimization, interaction analysis and desorption studies for the azo dyes adsorption onto chitosan films. Journal of Colloid and Interface Science, 411, 27-33.

WESKA, R. F., MOURA, J. M., BATISTA, L. M., RIZZI, J., PINTO, L. A. A. (2007), Optimization of deacetylation in the production of chitosan from shrimp wastes: Use of response surface methodology. Journal of Food Engineering, 80, 749-753.

WIBOWO, A., VELAZQUEZ, G., SAVANT, V., TORRES, J. A. (2007), Effect of chitosan type on proteins and water recovery efficiency from surimi wash water treated with chitosan-alginate complexes. Bioresource Technology, 98 (3), 539 - 545.

\section{AGRADECIMENTOS}

Os autores agradecem a FURG, CAPES e CNPq. 